\title{
INVESTIGACIONES
}

\section{0 años de Estudios Pedagógicos: análisis bibliométrico}

\author{
40 years of Estudios Pedagógicos: bibliometric analysis
}

\author{
Mikel Pérez-Gutiérrez ${ }^{a, b}$, Roberto Iván Lagos-Hernández \\ ${ }^{a}$ Departamento de Educación. Universidad de Cantabria, Santander, España. \\ mikel.perez@unican.es \\ ${ }^{b}$ Grupo de investigación de "Estudios Documentales en Ciencias de la Actividad Física \\ y de la Educación" de la Universidad de León. \\ ${ }^{\mathrm{c}}$ Facultad de Educación. Universidad Autónoma de Chile, Temuco, Chile. \\ roberto.lagos@uautonoma.cl
}

\begin{abstract}
RESUMEN
El objetivo del trabajo fue realizar un análisis bibliométrico de los artículos publicados en Estudios Pedagógicos desde 1976 hasta 2015. Para esto, se revisaron todos los números publicados recogiendo la información bibliográfica básica junto con la afiliación, tipo de documento, década y temática de cada documento en el programa Endnote X6 y posteriormente exportados al Excel 2013 para calcular los aspectos de productividad, materias y patrones de colaboración. En total, se publicaron 700 documentos, primando las investigaciones $(69,43 \%)$, dedicadas al estudio de las Ciencias de la educación y ambiente educacional (20,71\%), participando 1087 autores de 188 instituciones diferentes y 21 países, y observándose un incremento de la colaboración a lo largo del tiempo. En conclusión, Estudios Pedagógicos ha contribuido a la difusión de una cantidad considerable de trabajos científicos relacionados con la investigación educacional, principalmente de aquellos desarrollados en la propia Universidad Austral de Chile y en su mismo país.
\end{abstract}

Palabras claves: bibliología, publicación científica, historia, Ciencias de la Educación, Chile.

\begin{abstract}
The aim of this paper was to carry out a bibliometric analysis of the articles published in Estudios Pedagógicos journal from 1976 to 2015. Thus, all published issues were checked, collecting the basic bibliographic information as well as the affiliation, type of document, decade and subject of each document within Endnote X6 programme and then exported to Excel 2013 for determining productivity, subjects and collaboration patterns. In total, 700 documents were published, predominating researches $(69,43 \%)$, dedicated to Education Sciences and educational environment (20,71\%), participating 1087 authors from 188 different institutions and 21 countries, and depicting a rise in the collaboration along time. In conclusion, Estudios Pedagógicos has contributed to the dissemination of a considerable amount of scientific works related to educational research, mainly those developed by the own Universidad Austral de Chile and in their own country.
\end{abstract}

Key words: bibliology, scientific publications, history, Educational Sciences, Chile. 


\section{INTRODUCCIÓN}

La Universidad Austral de Chile fue fundada el 7 de septiembre de 1954 en la ciudad de Valdivia con la intención de responder a las necesidades educativas universitarias del sur del país (Universidad Austral de Chile, 2017), con el objetivo de "promover y desarrollar las funciones propias de una universidad" (citado en Almonacid Zapata, 2004, p. 166) y estructurada como las universidades clásicas europeas. Con esta idea en mente, se manifestó la creación de las facultades de Bellas Artes, Filosofía y Educación y Medicina en 1955, 1957 y 1959 respectivamente (Almonacid Zapata, 2004). Sin embargo, tras recibir distintas denominaciones, la Facultad de Filosofía y Educación pasó a llamarse de Filosofía y Humanidades en 1973 -tras la entrada ese mismo año del gobierno militar-, nombre que mantiene hasta la actualidad (Molina Verdejo, 2016).

En el contexto del gobierno militar (1973-1990) se sucedieron notables cambios sociales, económicos, políticos y culturales, como por ejemplo la violación de los derechos humanos, la implantación del modelo económico neoliberal, la división del país en 13 regiones administrativas o la reforma del sistema sanitario y educativo (Collier y Sater, 1999; Concha Cruz y Maltés Cortés, 2001; Silva Galdames, 1995). Dichos cambios tuvieron una profunda repercusión en el ámbito universitario, sucediéndose tres etapas que se iniciaron con la intervención militar y la eliminación de autoridades, organizaciones y centros universitarios (1973-1979), continuó con la implantación de un nuevo modelo universitario que impulsaba el sector privado y la competencia (1979-1981) y finalizó con la caída del modelo económico social y universitario (Garretón y Martínez, 1985; Núñez Prieto, 1984). Es en dicho contexto cuando aparece la revista Estudios Pedagógicos.

Dicha publicación inició su andar en el año 1976 bajo la dirección de Erwin Haverbeck Ojeda, quien además era decano de la Facultad de Letras y Educación (Almonacid Zapata, 2004; Molina Verdejo, 2016), convirtiéndose en una de las revistas científicas más antiguas de Chile relacionadas con la Educación, ya que la revista titulada Pensamiento Educativo, Revista de Investigación Latinoamericana (PEL) fue publicada por primera vez en 1993 bajo dicho nombre pero es heredera de los Anales de la Facultad de Filosofía y Ciencias de la Educación de la Pontificia Universidad Católica de Chile que comenzó a editarse en 1953 (Pontificia Universidad Católica de Chile, 2017). Con posterioridad a estas, se han editado un amplio conjunto de revistas educativas como son Perspectiva Educacional en 1980 (Pontificia Universidad Católica de Valparaíso, 2017), Paideia en 1990 aproximadamente (Universidad de Concepción, 2017), Calidad en la Educación en 1993 (Consejo Nacional de Educación, 2017), Docencia en 1996 (Docencia, 2017), Temas Pedagógicos en 1996 (Dialnet, 2017), Enfoques Educacionales en 1998 (Universidad de Chile, 2017), Umbral 2000 en 1999 (Latindex, 2017), Diálogos Educativos en 2001 (Diálogos Educativos, 2017), Revista de Estudios y Experiencias en Educación en 2001 (Revista de Estudios y Experiencias en Educación, 2017), Revista Chilena de Educación Científica en 2001 (Revista Chilena de Educación Científica, 2017), Revista de Educación en Ciencias de la Salud en 2004 (Revista de Educación en Ciencias de la Salud, 2017), Formación Universitaria en 2008 (Centro de Información Tecnológica, 2017) e Infancia, Educación y Aprendizaje en 2015 (Universidad de Valparaíso, 2017). Respecto a la relevancia de estas revistas, solo Estudios Pedagógicos y Formación Universitaria han conseguido ser indexadas en Scielo-Chile y SCOPUS, mientras que Calidad en la Educación también forma parte de Scielo-Chile, lo que implica una mayor visibilización de su impacto 
disciplinario y un interés por producir análisis respecto de los contenidos que se abordan en dichas publicaciones.

Por otra parte, la bibliometría, definida como la aplicación de técnicas estadísticas a la información bibliográfica (De Bellis, 2009; Diodato, 2012), se ha convertido en una herramienta fundamental para analizar la evolución y comportamiento de la información debido a su progresivo incremento durante las últimas décadas (Zulueta, 2011). En el ámbito científico, los estudios bibliométricos son fundamentales para conocer la evolución de la ciencia, detectar fortalezas y debilidades de la investigación, identificar patrones en la comunicación científica (Moya-Anegón, 2014) y mejorar la gestión de las inversiones en ciencia y tecnología (Thomson Reuters, 2008).

Dentro del campo educativo, la bibliometría también ha sido aplicada para determinar los patrones de publicación de sus profesionales (Hernández González, Reverter Masià, Jové Deltell y Mayolas Pi, 2013; Reverter Masià, Hernández González, Jové Deltell y Legaz Arrese, 2013), para evaluar el comportamiento y calidad de la investigación producida en distintos continentes y países como Asia (Jung y Horta, 2013), Australia (Phelan, Anderson y Bourke, 2000), Filipinas (Vinluan, 2012), Europa (Calderhead, 1997), Suiza (Diem y Wolter, 2013), Iberoamérica (Brunner Ried y Salazar Muñiz, 2012), México (Arenas, Valles y Arenas, 2000), Cuba (Arencibia Jorge, 2010), Brasil (Campos y Favero, 1994; Coutinho et al., 2012; Hayashi, Ferreira Jr., Bitar, Hayashi y da Silva, 2008) o España (Moya-Anegón, 2003) así como analizar el comportamiento de distintos grupos de revistas educativas (Anta Cabreros, 2007; Fuentes, Luque y López Gómez, 2012; García Sánchez, Rodríguez Pérez y González Sánchez, 2005; Maz-Machado, Jiménez-Fanjul y Adamuz-Povedano, 2014; Ruiz Corbella, Galán González y Diestro Fernández, 2014; Togia y Tsigilis, 2006; Torres Verdugo y Rovalo de Robles, 2015; Vanderstraeten, 2011; Zawacki-Richter, Anderson y Tuncay, 2010).

Finalmente, la bibliometría ha sido aplicada recientemente al estudio de distintas revistas educativas como Aula Abierta (Granados, Ariza, Gómez-García y Ramiro, 2011), Bordón (Gutiérrez-Arenas, 2012; Zich, 2011), Educación de la Pontificia Universidad Católica del Perú (Blanco Olea, 2008), Educación XX1 (Gómez-García, Ramiro, Ariza y Granados, 2012), Revista Complutense de Educación (Carpallo Bautista y Burgos Bordonau, 2008), Revista Iberoamericana de Educación Superior (Osorio-Madrid, 2014) o Revista de Investigación Educativa (Ariza y Quevedo-Blasco, 2013) para evaluar la trayectoria y situación de dichas publicaciones (Ariza y Quevedo-Blasco, 2013; Blanco Olea, 2008; Carpallo Bautista y Burgos Bordonau, 2008; Gutiérrez-Arenas, 2012), conocer su aporte al campo educativo (Osorio-Madrid, 2014; Zich, 2011) y mejorar su visibilidad e impacto (Gómez-García et al., 2012; Granados et al., 2011). Estos trabajos han centrado su interés en el análisis de los artículos que se han publicado y su distribución por año, autoría, instituciones, países y temas, la colaboración entre autores, el factor de impacto de sus artículos, los patrones de citación o su visibilidad, convirtiéndose en aportes fundamentales para el estudio de una parte de la actividad científica en este campo de estudio (Calatayud Soler y Sala Such, 1992). Por esto, el objetivo del presente trabajo fue realizar un análisis bibliométrico de los artículos publicados en la revista Estudios Pedagógicos desde su origen en 1976 hasta el año 2015 inclusive. Dicho análisis está centrado en el estudio de la productividad, materias y colaboración con el propósito de ofrecer una visión general de la aportación de la revista a la investigación educativa en Chile y además con la convicción de que se constituye en una aproximación histórica al desarrollo de este campo de conocimiento durante los últimos 40 años. 


\section{METODOLOGÍA}

\subsection{OBJETO DE ESTUDIO}

Se incluyeron todos los documentos publicados en la revista Estudios Pedagógicos desde su inicio en 1976 hasta el año 2015 inclusive, que tuvieran un carácter investigativo (artículos, ensayos, investigaciones, ponencias y revisiones). Los informes, editoriales, discursos, notas, documentos y reseñas no se incluyeron en la presente investigación debido a su carácter informativo.

\subsection{RECOGIDA DE INFORMACIÓN}

Para la recogida de información se consultaron todos los números publicados por la revista, en formato papel para aquellos editados entre 1976 y 1996 y en formato digital para los editados entre 1997 y 2015 a través de la web Scielo. La información de cada documento fue introducida en una ficha bibliográfica creada en el programa gestor de referencias Endnote X6. Se registraron los datos bibliográficos básicos (autor, año, título, revista, volumen, número, páginas) de cada documento de acuerdo con las recomendaciones de la Agencia Española de Normalización (2013). Además, se anotó la afiliación del autor, el tipo de documento, la década de publicación de acuerdo a la historia de la revista y la temática de cada documento. Para la clasificación temática se revisó el título, palabras clave y resumen de cada documento, determinando su inclusión en alguna de las 14 temáticas principales propuestas en el tesauro de la United Nations Educational, Scientific and Cultural Organization (UNESCO), en consideración a su especialidad en el ámbito educativo, su carácter internacional, su grado de actualización (United Nations Educational, Scientific and Cultural Organization, 2016) y su aplicación en estudios similares (Blanco Olea, 2008).

\subsection{ANÁLISIS DE DATOS}

La información recopilada fue exportada al programa Excel 2013 para su tabulación y análisis mediante estadística descriptiva. El análisis bibliométrico de productividad y materias estuvo centrado en el análisis de frecuencias y porcentajes para determinar la producción de artículos por año, década, tipo de documento, temática, institución y país de afiliación del autor. Para el análisis de colaboración se contabilizó la cantidad total de artículos (AT), los artículos individuales (AI), artículos realizados en coautoría (AC), el promedio de autores por artículo, así como el porcentaje de colaboración (\%C), utilizando para ello la fórmula propuesta por Valenciano Valcárcel, Devís Devís, Villamón y Peiró Velert (2010) siendo el \%C=AC/AT.

\section{RESULTADOS}

La cantidad total de documentos asciende a 700 (Tabla 1), primando las investigaciones $(69,43 \%)$ seguidas por los ensayos $(13,71 \%)$. Su evolución temporal muestra una tendencia creciente siendo la década 2006-2015 la que reúne un 59,86\% del total de documentos publicados. 
Tabla 1. Distribución del tipo de documento por década

\begin{tabular}{|c|c|c|c|c|c|c|c|c|c|c|}
\hline \multirow{2}{*}{$\begin{array}{c}\text { Tipo de } \\
\text { documento }\end{array}$} & \multicolumn{2}{|c|}{ Total } & \multicolumn{2}{|c|}{ 1976-1985 } & \multicolumn{2}{|c|}{ 1986-1995 } & \multicolumn{2}{|c|}{ 1996-2005 } & \multicolumn{2}{|c|}{ 2006-2015 } \\
\hline & $\mathbf{n}$ & $\%$ & $\mathrm{n}$ & $\%$ & $\mathbf{n}$ & $\%$ & n & $\%$ & $\mathbf{n}$ & $\%$ \\
\hline Artículos & 33 & 4,71 & 27 & 3,86 & 6 & 0,86 & & & & \\
\hline Ensayos & 96 & 13,71 & & & 19 & 2,71 & 45 & 6,43 & 32 & 4,57 \\
\hline Investigaciones & 486 & 69,43 & 58 & 8,29 & 61 & 8,71 & 62 & 8,86 & 305 & 43,57 \\
\hline Ponencias & 3 & 0,43 & 2 & 0,29 & 1 & 0,14 & & & & \\
\hline Revisiones & 82 & 11,71 & & & & & & & 82 & 11,71 \\
\hline Total & 700 & 100 & 87 & 12,43 & 87 & 12,43 & 107 & 15,29 & 419 & 59,86 \\
\hline
\end{tabular}

Los documentos fueron clasificados en 13 de los 14 temas propuestos por la UNESCO dentro del ámbito educativo, destacando las ciencias de la educación y ambiente educacional $(20,71 \%)$. En un segundo grupo de temáticas se encuentran las materias de enseñanza básica y general $(15,71 \%)$, enseñanza y formación $(13,86)$, evaluación de la educación $(12,71)$, materias de enseñanza técnica y profesional $(10,43)$ y política educacional $(9 \%)$, mientras que el resto de los temas no superan el $5 \%$.

La distribución por década de estas temáticas muestra la evolución temporal de los intereses de los investigadores, siendo las ciencias de la educación y ambiente educacional un tema principal en todas ellas. Con excepción de este tema principal, la temática más destacada en la década de 1976-1985 fue materias de enseñanza básica y general (3,43\%), mientras que en las décadas 1986-1995 y 1996-2005 fue enseñanza y formación (2,14\% y $3,14 \%$ respectivamente).

Tabla 2. Distribución de las temáticas presentes en los artículos por década

\begin{tabular}{|c|c|c|c|c|c|c|c|c|c|c|}
\hline \multirow{2}{*}{ Temática } & \multicolumn{2}{|c|}{ Total } & \multicolumn{2}{|c|}{ 1976-1985 } & \multicolumn{2}{|c|}{ 1986-1995 } & \multicolumn{2}{|c|}{ 1996-2005 } & \multicolumn{2}{|c|}{ 2006-2015 } \\
\hline & $\mathbf{n}$ & $\%$ & $\mathbf{n}$ & $\%$ & $\mathbf{n}$ & $\%$ & $\mathbf{n}$ & $\%$ & $\mathbf{N}$ & $\%$ \\
\hline $\begin{array}{l}\text { Administración de la } \\
\text { educación }\end{array}$ & 12 & 1,71 & 2 & 0,29 & 2 & 0,29 & & & 8 & 1,14 \\
\hline $\begin{array}{c}\text { Ciencias de la } \\
\text { educación y ambiente } \\
\text { educacional }\end{array}$ & 145 & 20,71 & 16 & 2,29 & 16 & 2,29 & 26 & 3,71 & 87 & 12,43 \\
\hline Enseñanza y formación & 97 & 13,86 & 10 & 1,43 & 15 & 2,14 & 22 & 3,14 & 50 & 7,14 \\
\hline $\begin{array}{c}\text { Evaluación de la } \\
\text { educación }\end{array}$ & 89 & 12,71 & 17 & 2,43 & 13 & 1,86 & 14 & 2,00 & 45 & 6,43 \\
\hline $\begin{array}{l}\text { Gestión de la } \\
\text { educación }\end{array}$ & 14 & 2,00 & 1 & 0,14 & 1 & 0,14 & 4 & 0,57 & 8 & 1,14 \\
\hline $\begin{array}{l}\text { Instalaciones y } \\
\text { recursos educativos }\end{array}$ & 18 & 2,57 & 6 & 0,86 & 3 & 0,43 & & & 9 & 1,29 \\
\hline
\end{tabular}




\begin{tabular}{|c|c|c|c|c|c|c|c|c|c|c|}
\hline $\begin{array}{c}\text { Materias de enseñanza } \\
\text { básica y general }\end{array}$ & 110 & 15,71 & 24 & 3,43 & 8 & 1,14 & 13 & 1,86 & 65 & 9,29 \\
\hline $\begin{array}{c}\text { Materias de enseñanza } \\
\text { técnica y profesional }\end{array}$ & 73 & 10,43 & 2 & 0,29 & 2 & 0,29 & 6 & 0,86 & 63 & 9,00 \\
\hline Planes de estudios & 29 & 4,14 & 4 & 0,57 & 9 & 1,29 & 1 & 0,14 & 15 & 2,14 \\
\hline $\begin{array}{c}\text { Planificación de la } \\
\text { educación }\end{array}$ & 13 & 1,86 & & & 4 & 0,57 & 2 & 0,29 & 7 & 1,00 \\
\hline Política educacional & 63 & 9,00 & 2 & 0,29 & 4 & 0,57 & 11 & 1,57 & 46 & 6,57 \\
\hline $\begin{array}{c}\text { Profesionales de la } \\
\text { educación }\end{array}$ & 5 & 0,71 & 1 & 0,14 & 3 & 0,43 & & & 1 & 0,14 \\
\hline $\begin{array}{c}\text { Sistemas y niveles de } \\
\text { enseñanza }\end{array}$ & 32 & 4,57 & 2 & 0,29 & 7 & 1,00 & 8 & 1,14 & 15 & 2,14 \\
\hline Total & $\mathbf{7 0 0}$ & $\mathbf{1 0 0}$ & $\mathbf{8 7}$ & $\mathbf{1 2 , 4 3}$ & $\mathbf{8 7}$ & $\mathbf{1 2 , 4 3}$ & $\mathbf{1 0 7}$ & $\mathbf{1 5 , 2 9}$ & $\mathbf{4 1 9}$ & $\mathbf{5 9 , 8 6}$ \\
\hline
\end{tabular}

La cantidad de autores, instituciones y países representados en la revista Estudios Pedagógicos distribuidos por década muestra una tendencia creciente a lo largo del tiempo, con excepción del número de autores de la década 1996-2005, donde es posible apreciar cifras más altas durante el último periodo analizado.

Tabla 3. Cantidad y porcentaje de artículos, autores, instituciones y países distribuidos por década

\begin{tabular}{|c|c|c|c|c|c|c|}
\hline \multirow{2}{*}{ Década } & \multicolumn{2}{|c|}{ Autores } & \multicolumn{2}{c|}{ Instituciones } & \multicolumn{2}{c|}{ Países } \\
\cline { 2 - 7 } & $\mathbf{n}$ & $\mathbf{\%}$ & $\mathbf{n}$ & $\mathbf{\%}$ & $\mathbf{n}$ & $\mathbf{\%}$ \\
\hline $1976-1985$ & 120 & 11,04 & 9 & 4,79 & 2 & 9,52 \\
\hline $1986-1995$ & 134 & 12,33 & 27 & 14,36 & 5 & 23,81 \\
\hline $1996-2005$ & 128 & 11,78 & 43 & 22,87 & 15 & 71,43 \\
\hline $2006-2015$ & 785 & 72,22 & 156 & 82,98 & 18 & 85,71 \\
\hline Total & $\mathbf{1 0 8 7}$ & $\mathbf{1 0 0}$ & $\mathbf{1 8 8}$ & $\mathbf{1 0 0}$ & $\mathbf{2 1}$ & $\mathbf{1 0 0}$ \\
\hline
\end{tabular}

n: frecuencia; \%: respecto al total de autores (1087), instituciones (188) y países (21).

Tal como se aprecia en la Tabla 4, la cantidad de artículos individuales y en coautoría por década aumentaron irregularmente a lo largo del periodo estudiado. Con excepción de la década 1996-2005, tanto el promedio de autores por artículo como el porcentaje de colaboración también aumentaron con el tiempo, mostrando un incremento de la colaboración entre los investigadores que publicaron en Estudios Pedagógicos. 
Tabla 4. Cantidad de artículos individuales, en coautoría, promedio de autores por artículos y porcentaje de colaboración distribuidos por década

\begin{tabular}{|c|c|c|c|c|}
\hline Década & $\begin{array}{c}\text { Artículos } \\
\text { individuales }\end{array}$ & $\begin{array}{c}\text { Artículos en } \\
\text { coautoría }\end{array}$ & $\begin{array}{c}\text { Promedio } \\
\text { autores/artículo }\end{array}$ & $\begin{array}{c}\text { \% de } \\
\text { colaboración }\end{array}$ \\
\hline $1976-1985$ & 59 & 28 & 2,01 & 32,18 \\
\hline $1986-1995$ & 44 & 43 & 2,23 & 49,43 \\
\hline $1996-2005$ & 69 & 38 & 1,66 & 35,51 \\
\hline $2006-2015$ & 139 & 280 & 2,45 & 66,83 \\
\hline Total & $\mathbf{3 1 1}$ & $\mathbf{3 8 9}$ & $\mathbf{2 , 2 5}$ & $\mathbf{5 5 , 5 7}$ \\
\hline
\end{tabular}

La distribución de firmas por institución (Tabla 5) mostró la predominancia de los trabajos desarrollados en la Universidad Austral de Chile $(22,86 \%)$, con más del doble de representación que la Pontificia Universidad Católica de Chile (9,02\%), mientras que el resto de instituciones no superaron el $8 \%$ del total de firmas respectivamente.

Tabla 5. Distribución de firmas por institución

\begin{tabular}{|l|c|c|}
\hline Institución & $\mathbf{n}$ & $\mathbf{\%}$ \\
\hline Universidad Austral de Chile & 360 & 22,86 \\
\hline Pontificia Universidad Católica de Chile & 142 & 9,02 \\
\hline Universidad de Concepción & 117 & 7,43 \\
\hline Pontificia Universidad Católica de Valparaíso & 69 & 4,38 \\
\hline Universidad de Chile & 66 & 4,19 \\
\hline Universidad de La Frontera & 52 & 3,30 \\
\hline Desconocida & 50 & 3,17 \\
\hline Universidad de Talca & 36 & 2,29 \\
\hline Universidad Diego Portales & 33 & 2,10 \\
\hline Universidad Metropolitana de Ciencias de la Educación & 32 & 2,03 \\
\hline Universidad Católica de Temuco & 27 & 1,71 \\
\hline Universidad de Playa Ancha & 24 & 1,52 \\
\hline Universidad Nacional del Comahue (Argentina) & 24 & 1,52 \\
\hline Universidad Alberto Hurtado & 22 & 1,40 \\
\hline Universidad de Atacama & 18 & 1,14 \\
\hline Otras & 503 & 31,94 \\
\hline Total & $\mathbf{1 5 7 5}$ & $\mathbf{1 0 0}$ \\
\hline
\end{tabular}

n: frecuencia; \%: respecto al total de firmas (1575). 
Por último, Estudios Pedagógicos incluyó fundamentalmente los trabajos desarrollados en Chile $(78,54 \%)$ seguidos por aquellos realizados en España $(6,86 \%)$. El resto de los países con mayor representación en la revista fueron del ámbito latinoamericano.

Tabla 6. Distribución de firmas por país

\begin{tabular}{|c|c|c|}
\hline País & $\mathbf{n}$ & \% \\
\hline Chile & 1237 & 78,54 \\
\hline España & 108 & 6,86 \\
\hline Desconocido & 50 & 3,17 \\
\hline Colombia & 49 & 3,11 \\
\hline Argentina & 48 & 3,05 \\
\hline Brasil & 33 & 2,10 \\
\hline México & 12 & 0,76 \\
\hline Otros & 38 & 2,41 \\
\hline Total & $\mathbf{1 5 7 5}$ & $\mathbf{1 0 0}$ \\
\hline
\end{tabular}

$\mathrm{n}$ : frecuencia; \%: respecto al total de firmas (1575).

\section{DISCUSIÓN}

La revista Estudios Pedagógicos ha publicado un total de 700 trabajos en los 40 años de historia que fueron sometidos a análisis, siendo el 59,86\% de ellos publicados en el periodo 2006-2015. Además, ha contado con la colaboración de 1087 autores pertenecientes a 188 instituciones de 21 países diferentes. La temática de estos trabajos ha estado centrada en ciencias de la educación y ambiente educacional, aunque también hay una representación destacada de otras cinco temáticas, cuyo porcentaje oscila entre un $9 \%$ y un $15,71 \%$. El predominio de artículos individuales (59 vs 28) y un bajo porcentaje de colaboración $(32,18 \%)$ durante su primera década de publicación contrastan con el incremento de los artículos en coautoría (280) y la colaboración $(66,83 \%)$ del último periodo analizado. Finalmente, la Universidad Austral de Chile fue la institución con mayor porcentaje de firmas $(22,86 \%)$ mientras que Chile fue el país más representado $(78,54 \%)$.

La aportación de la revista Estudios Pedagógicos al ámbito educativo dentro de Chile no sólo destaca por su amplia historia, siendo una de las revistas académicas dedicada al ámbito educativo más antigua de Chile al publicarse por primera vez en 1976, sino también por la cantidad y variedad de documentos científicos que ha publicado. Aunque las investigaciones han sido la principal tipología de documentos, también es destacable la aparición de una cantidad considerable de revisiones y ensayos, por encima del 10\% del total respectivamente, mostrando la apertura editorial de la revista para reunir los distintos productos derivados de la actividad investigadora y/o reflexiva de los estudiosos del campo educativo. La distribución de esta producción científica a lo largo de las décadas muestra el 
crecimiento de la investigación educativa durante el periodo 2006-2015, con la publicación del 59,86\% del total de documentos, mostrando el mismo patrón que la evolución de la ciencia en Chile (Moya-Anegón, 2015). De esta manera, Estudios Pedagógicos no solo se ha convertido en uno de los principales medios de difusión de las investigaciones educativas en Chile, afianzando su posición durante el último periodo analizado con 419 documentos publicados, sino que también representa la evolución de este campo de conocimiento y el interés social y académico por esta temática.

La variedad temática de los contenidos publicados en Estudios Pedagógicos también es una de sus principales características, encontrándose representados la mayoría de los temas propuestos por la UNESCO, aunque destacan aquellos documentos relacionados con las Ciencias de la educación y ambiente educacional, ya que esta temática está compuesta por las diferentes disciplinas científicas que sostienen teóricamente al campo educativo y por el estudio del contexto educativo respectivamente. Además, otras cinco temáticas reúnen entre el $9 \%$ y $15 \%$ de los documentos, encontrándose relacionadas con las diferentes materias o asignaturas que componen la enseñanza en sus distintos niveles, los procesos de enseñanza-aprendizaje, la evaluación o las políticas educacionales aplicadas en el aula. Por el contrario, el estudio relacionado con los profesionales de la educación $(0,71 \%)$ o la administración de la educación $(1,71 \%)$ apenas aparecen representados en la revista. Se pudo apreciar que la investigación relativa a los establecimientos de enseñanza (escuelas, centros de formación, instituciones de enseñanza o universidades) fue nula. Por lo tanto, parece que la preocupación de la actividad investigadora está principalmente centrada en la labor educativa al interior del aula, en vez de aquellos procesos vinculados a los aspectos administrativos (administración de la educación, gestión de la educación, instalaciones y recursos educativos, planificación de la educación), curriculares (planes de estudios) o los diferentes actores educativos (profesionales de la educación). Aunque la distribución por décadas de dichas temáticas es irregular, la mayoría muestra una tendencia creciente a lo largo del tiempo, con excepción de algunos temas que no aparecen en determinados periodos o los estudios relativos a los profesionales de la educación que mantuvieron su frecuencia. De esta manera, Estudios Pedagógicos ha mostrado a lo largo de los años una gran variedad temática, dando cabida a estudios relacionados con cualquier aspecto educativo, aunque en distinta proporción, cumpliendo con su objetivo de ser "una revista científica inter transdisciplinaria en Ciencias de la Educación" (Estudios Pedagógicos, 2017). Sin embargo, determinadas áreas temáticas están poco o nada representadas en sus contenidos, por lo que debería intentar captar los trabajos realizados por los académicos especialistas en estas áreas para ampliar las aproximaciones teóricas a las mismas y así fortalecer su carácter transdisciplinar.

Por otra parte, Estudios Pedagógicos se ha convertido en un medio de difusión de los trabajos de una cantidad creciente de autores, instituciones y países, siendo el periodo 20062015 en el que la revista ha consolidado su posición dentro del campo educativo chileno al contar con 785 autores de 156 instituciones diferentes. Además, la participación de autores de 18 países distintos representa la proyección internacional que busca esta publicación.

Respecto a la colaboración, durante el periodo 1976-1985 predominaron los artículos individuales frente a los realizados en coautoría (59 vs 28) estando el porcentaje de colaboración en el 32,18\%. Esta situación se ha visto revertida en el periodo 2006-2015 con el predominio de los artículos en coautoría (280) frente a los individuales (139) y el aumento del porcentaje de colaboración hasta el 66,83\%. De esta manera, Estudios Pedagógicos 
refleja la tendencia actual de la investigación educativa a realizarse en colaboración con otros académicos, debido a la creciente complejidad e interdisciplinariedad de la ciencia durante las últimas décadas (Beaver y Rosen, 1979; Lee y Bozeman, 2005; Luukkonen, Persson y Sivertsen, 1992). Sin embargo, la estructura de las redes de colaboración no ha variado sustancialmente a lo largo de estas cuatro décadas de historia, ya que se ha pasado de 2,01 autores por artículo en 1976-1985 a 2,45 autores por artículo, es decir, sigue predominando el trabajo en parejas para desarrollar la investigación educacional.

La distribución de firmas por institución mostró que la revista Estudios Pedagógicos ha sido principalmente un medio de difusión de las investigaciones realizadas en la propia Universidad Austral de Chile además de las investigaciones realizadas dentro del ámbito nacional, ya que 13 de las 15 instituciones con mayor cantidad de firmas son chilenas, cumpliendo el objetivo primigenio de la revista (Haverbeck Ojeda, 1976). Estos resultados son corroborados en la Tabla 6, siendo el 78,54\% de las firmas chilenas. También es destacable su visibilidad internacional con 21 países representados en total y su red de colaboración con 188 instituciones diferentes, aunque el aporte total de países extranjeros apenas roza el $20 \%$. Por esto, tras el proceso de consolidación que ha sufrido la revista durante el último periodo analizado, en relación a la producción científica chilena, deberían plantearse estrategias que amplíen y fortalezcan la proyección internacional iniciada por la revista para convertirse en medio de difusión de los trabajos de investigación educativa provenientes de Iberoamérica, tal como señala la línea editorial más reciente de la revista (Estudios Pedagógicos, 2017).

\section{CONCLUSIONES}

La revista Estudios Pedagógicos, en sus 40 años de historia, ha contribuido a la difusión de una cantidad considerable de trabajos científicos relacionados con la investigación educacional, principalmente de aquellos desarrollados en la propia Universidad Austral de Chile y los realizados en Chile, cumpliendo el objetivo explicitado en la primera edición de 1976 de apertura editorial, fundamentalmente durante la última década analizada. Además, esta publicación ha confirmado el carácter inter transdisciplinar que ha pretendido poseer a lo largo de este tiempo, incluyendo artículos relacionados con la mayoría de las áreas temáticas propuestas en el tesauro de la UNESCO, aunque en proporciòn desigual. En este sentido, seis áreas temáticas agruparon un $82,43 \%$ del total de trabajos publicados, siendo el estudio relacionado con las ciencias de la educación y el ambiente educacional la principal de ellas. Por lo tanto, Estudios Pedagógicos requiere fortalecer aquellas áreas temáticas menos representadas para ampliar su variedad de contenidos.

A pesar de la apertura editorial que presenta la revista con la cantidad de autores, instituciones y países representados en sus páginas, destacan las aportaciones nacionales y especialmente de la Universidad Austral de Chile. Una vez que Estudios Pedagógicos se ha consolidado como medio de difusión de las investigaciones educacionales en Chile, fundamentalmente durante el periodo 2006-2015, se requiere la implantación de políticas editoriales que favorezcan la colaboración de los investigadores chilenos con académicos iberoamericanos para ampliar la cobertura territorial de sus contenidos, fortalecer sus redes de colaboración, aumentar su visibilidad y disminuir la cantidad de aportaciones realizadas desde la propia Universidad Austral de Chile para evitar la endogamia editorial. 
Además, la predominancia de trabajos realizados individualmente durante el periodo 1976-1985 con una tasa del 32,18\% de colaboración contrasta con el aumento del número de artículos realizados en coautoría durante el periodo 2006-2015 alcanzando hasta el 66,83\% de colaboración. A pesar de este aumento de los trabajos desarrollados en coautoría, las redes de colaboración apenas han variado ligeramente su estructura, al seguir predominando los grupos de investigación formados por dos académicos.

Finalmente, a través del análisis bibliométrico de la revista Estudios Pedagógicos se ha ofrecido una visión parcial de la evolución de la investigación educacional desarrollada en Chile así como sus características, fortalezas y debilidades, siendo necesario el estudio del resto de revistas científico-técnicas del ámbito educativo chileno para completar la visión general y la aproximación histórica a este campo de conocimiento. Futuras investigaciones deberían analizar los patrones de citación de los artículos publicados en la revista para conocer los autores y artículos más importantes, así como los tipos de documentos que han fundamentado estos trabajos.

\section{REFERENCIAS BIBLIOGRÁFICAS}

Agencia Española de Normalización (2013). UNE-ISO 690-2013. Información y documentación. Directrices para la redacción de referencias bibliográficas y de citas de recursos de información. Madrid: Agencia Española de Normalización.

Almonacid Zapata, F. (2004). Historia de la Universidad Austral de Chile (1954-2003): su historia en el contexto universitario nacional. Santiago de Chile: Universidad Austral de Chile.

Anta Cabreros, C. (2007). Análisis bibliométrico de la investigación educativa divulgada en publicaciones periódicas españolas entre 1990-2002. Revista Electrónica de Investigación Educativa, 10(1), 1-17.

Arenas, J. L. d., Valles, J., \& Arenas, M. (2000). Educational research in Mexico: Socio-demographic and visibility issues. Educational Research, 42(1), 85-90.

Arencibia Jorge, R. (2010). Visibilidad Internacional de la Ciencia y Educación Superior Cubanas: desafíos del estudio de la producción científica (Tesis para optar al grado de Doctor en Documentación), Universidad de Granada, Universidad de La Habana, Granada, La Habana.

Ariza, T. \& Quevedo-Blasco, R. (2013). Análisis bibliométrico de la Revista de Investigación Educativa (2000-2012). Revista de Investigacion Educativa, 31(1), 31-52. doi: 10.6018/ rie.31.1.160321

Beaver, D. de B., \& Rosen, R. (1979). Studies in Scientific Collaboration. Part II. Scientific CoAuthorship, Research Productivity and Visibility in the French Scientific Elite, 1799-1830. Scientometrics, 1(2), 133-149.

Blanco Olea, F. S. (2008). Análisis bibliométrico de la revista "Educación" de la Pontificia Universidad Católica del Perú (1992-2005). (Título de Grado), Pontificia Universidad Católica del Perú, Lima, Perú. Recuperado de http://eprints.rclis.org/15297/1/BLANCO.pdf

Brunner Ried, J. J., \& Salazar Muñiz, F.A. (2012). Investigación educacional en Iberoamérica: entre la invisibilidad y la medición. Magis: Revista Internacional de Investigación en Educación, 4(9), 559-575.

Calatayud Soler, R., \& Sala Such, E. (1992). 50 años de vida de la Revista Española de Pedagogía: una aproximación bibliométrica (1943-1990). Revista Española de Pedagogía, 50(192), 271288.

Calderhead, J. (1997). La investigación educativa en Europa en los últimos diez años. Revista de Educación, (312), 9-20. 
Campos, M.M., \& Favero, O. (1994). A pesquisa em educação no Brasil. Cadernos de Pesquisa, (88), 5-17.

Carpallo Bautista, A., \& Burgos Bordonau, E. (2008). Estudio bibliométrico y de calidad de la Revista Complutense de Educación (1990-2007). Revista Complutense de Educación, 19(7), 13-29.

Centro de Información Tecnológica (2017). Formación Universitaria. Acerca de la revista. Recuperado de http://www.citrevistas.cl/a1c-formacion.htm

Collier, S., \& Sater, W. F. (1999). Historia de Chile, 1808-1994. Madrid: Cambridge University Press.

Concha Cruz, A., \& Maltés Cortés, J. (2001). Historia de Chile. Barcelona: Bibliográfica Internacional.

Consejo Nacional de Educación (2017). Revista Calidad en la Educación: Acerca de la Revista. Recuperado de http://www.cned.cl/public/secciones/SeccionRevistaCalidad/revista_calidad_ acerca_de.aspx

Coutinho, R. X., Dávila, E. S., dos Santos, W. M., Rocha, J.B.T., Souza, D. O. G., Folmer, V. \& Puntel, R.L. (2012). Brazilian scientific production in science education. Scientometrics, 92(3), 697-710. doi: 10.1007/s11192-012-0645-5

De Bellis, N. (2009). Bibliometrics and Citation Analysis: from the Science Citation Index to Cybermetrics. Lanham, Toronto, Plymouth: The Scarecrow Press.

Dialnet (2017). Temas pedagógicos. Recuperado de https://dialnet.unirioja.es/servlet/ revista?codigo $=7618$

Diálogos Educativos (2017). Revista Electrónica Diálogos Educativos no 1. Recuperado de http:// dialogoseducativos.cl/index.php/using-joomla/extensions/components/146-revista-n001indice

Diem, A., \& Wolter, S. C. (2013). The Use of Bibliometrics to Measure Research Performance in Education Sciences. Research in Higher Education, 54(1), 86-114. doi: 10.1007/s11162-0129264-5

Diodato, V. P. (2012). Dictionary of bibliometrics. New York: Routledge.

Docencia (2017). Quienes somos. Recuperado de http://www.revistadocencia.cl/quienes-somos/

Estudios Pedagógicos (2017). Nosotros. Recuperado de http://revistas.uach.cl/index.php/estped/ about

Fuentes, J. L., Luque, D., \& López Gómez, E. (2012). Análisis bibliométrico de las revistas españolas de educación incluidas en el Journal Citation Report. Producción científica y elementos controvertidos. Teoría de la Educación, 24(1), 183-217.

García Sánchez, J. N., Rodríguez Pérez, C., \& González Sánchez, L. (2005). Valoración de la difusión en doce revistas de educación en español: análisis del factor de impacto y otros índices bibliométricos. Aula Abierta, 85, 3-44.

Garretón, M. A. \& Martínez, J. (1985). Universidades chilenas: historia, reforma e intervención. Santiago: SUR.

Gómez-García, A., Ramiro, M. T., Ariza, T., \& Granados, M. R. (2012). Estudio bibliométrico de Educación XX1. Educación XX1, 15(1), 17-41.

Granados, M. R., Ariza, T., Gómez-García, A. \& Ramiro, M. T. (2011). Estudio Bibliométrico de Aula Abierta. Aula Abierta, 39(3), 97-110.

Gutiérrez-Arenas, M. P. (2012). Estudio de revistas científicas españolas: el caso de "Bordón. Revista de pedagogía” (1984-2008). (Tesis para optar al grado de Doctor en Educación), Universidad de Córdoba, Córdoba, España.

Haverbeck Ojeda, E. (1976). Palabras preliminares. Estudios Pedagógicos, (1), 5.

Hayashi, M. C. P. I., Ferreira Jr., A., Bitar, M., Hayashi, C.A.M., \& da Silva, M.R. (2008). História da educação brasileira: a produção científica na biblioteca eletrônica SCIELO. Educação \& Sociedade, 29(102), 181-211. doi: http://dx.doi.org/10.1590/S0101-73302008000100010

Hernández González, V., Reverter Masià, J., Jové Deltell, M. C. \& Mayolas Pi, M.d.C. (2013). Hábitos de publicación de profesores de los departamentos de didáctica de la expresión corporal 
y educación física de las universidades públicas españolas. European Journal of Human Movement, (30), 1-12.

Jung, J., \& Horta, H. (2013). Higher education research in Asia: A scientometric perspective. Higher Education Quarterly, 67(4), 398-419.

Latindex (2017). Umbral 2000. Recuperado de http://www.latindex.org/latindex/ficha?folio=13617

Lee, S., \& Bozeman, B. (2005). The Impact of Research Collaboration on Scientific Productivity. Social Studies of Science, 35(5), 673-702.

Luukkonen, T., Persson, O., \& Sivertsen, G. (1992). Understanding Patterns of International Scientific Collaboration. Science, Technology, \& Human Values, 17(1), 101-126.

Maz-Machado, A., Jiménez-Fanjul, N., \& Adamuz-Povedano, N. (2014). Spanish Journals of Education \& Educational Research in the JCR: A bibliometric analysis of the citations. Library Philosophy and Practice (e-journal), 1121.

Molina Verdejo, R. (2016). Universidad Austral de Chile. Facultad de Filosofía y Humanidades. Historia. Recuperado de http://humanidades.uach.cl/facultad/historia/

Moya-Anegón, F. (2003). Visibilidad internacional de la investigación española en Ciencias de la Educación. El caso de la didáctica de la matemática. En E. Castro Martínez (Ed.), Investigación en educación matemática: séptimo Simposio de la Sociedad Española de Investigación en Educación Matemática (pp. 13-24). Granada: Sociedad Española de Investigación en Educación Matemática.

Moya-Anegón, F.d. (2014). Indicadores bibliométricos de la actividad científica española 2011. Madrid: Fundación Española para la Ciencia y Tecnología.

Moya-Anegón, F.d. (2015). Principales indicadores cienciométricos de la actividad científica chilena 2013. Informe 2015. Santiago, Madrid, Viña del Mar: Altazor Ediciones.

Núñez Prieto, R. I. (1984). Las transformaciones de la educación bajo el régimen militar. Volumen 2. Santiago: Programa Interdisciplinario de Investigaciones en Educación.

Osorio-Madrid, J. R. (2014). La Revista Iberoamericana de Educación Superior (RIES): tres años contribuyendo al conocimiento de la educación superior en la región. Revista Iberoamericana de Educación Superior, 5(12), 118-135.

Phelan, T. J., Anderson, D. S. \& Bourke, P. (2000). Educational research in Australia: a bibliometric analysis. In Higher Education Division (Ed.), The Impact of Educational Research (pp. 573671). Canberra: Commonwealth of Australia.

Pontificia Universidad Católica de Chile (2017). Pensamiento Educativo, Revista de Investigación Educacional Latinoamericana: Historia de la Revista. Recuperadode http://pensamientoeducativo. uc.cl/index.php/pel/about/history

Pontificia Universidad Católica de Valparaíso (2017). Perspectiva Educacional. Acerca de la revista. Recuperado de http://www.perspectivaeducacional.cl/index.php/peducacional/about

Reverter Masià, J., Hernández González, V., Jové Deltell, M. C. \& Legaz Arrese, A. (2013). Indicadores de producción de los profesores de Educación Física y Didáctica de la Expresión Corporal en España en la Web of Science. Perspectivas em Ciência da Informação, 18(3), 3-23.

Revista Chilena de Educación Científica (2017). Índice Revistas. Recuperado de http://rchec.umce. cl/index.php/revistas/indice-revistas

Revista de Educación en Ciencias de la Salud (2017). Quienes somos. Recuperado de http://www2. udec.cl/ofem/recs/

Revista de Estudios y Experiencias en Educación (2017). Inicio. Recuperado de http://www.rexe.cl/ ojournal/index.php/rexe/

Ruiz Corbella, M., Galán González, A., \& Diestro Fernández, A. (2014). Las revistas científicas de Educación en España: evolución y perspectivas de futuro. Relieve: Revista ELectrónica de Investigación y EValuación Educativa, 20(2), art. M1. doi: 10.7203/relieve.20.2.4361.

Silva Galdames, O. (1995). Breve historia contemporánea de Chile. México D. F.: Fondo de Cultura Económica. 
Thomson Reuters (2008). Whitepaper using bibliometrics: a guide to evaluating research performance with citation data. Recuperado de http://ip-science.thomsonreuters.com/m/pdfs/325133 thomson.pdf

Togia, A. \& Tsigilis, N. (2006). Impact factor and education journals: a critical examination and analysis. International Journal of Educational Research, 45(6), 362-379. doi: 10.1016/j. ijer.2007.02.001

Torres Verdugo, M. A. \& Rovalo de Robles, M. L. (2015). Perfil de las Revistas Iberoamericanas sobre Educación Analizadas en el iresie. En J. Asenjo, O. Macías \& J.C. Toscano (Eds.), Memorias del Congreso Iberoamericano de Ciencia, Tecnología, Innovación y Educación. Buenos Aires: Organización de Estados Iberoamericanos.

United Nations Educational, Scientific and Cultural Organization (2016). Tesauro de la UNESCO. Recuperado de http://databases.unesco.org/thessp/

Universidad Austral de Chile (2017). Universidad Austral de Chile. Historia. Recuperado de http:// www.uach.cl/transparencia/quienes-somos/historia

Universidad de Concepción (2017). Paideia, revista de educación. Recuperado de http://www. revistapaideia.cl/index.php/PAIDEIA/index

Universidad de Chile (2017). Revista Enfoques Educacionales. Portada. Recuperado de http://www. revistas.uchile.cl/index.php/REE/index

Universidad de Valparaíso (2017). Infancia, Educación y Aprendizaje. Recuperado de http://revistas. uv.cl/index.php/IEYA

Valenciano Valcárcel, J., Devís Devís, J., Villamón, M. \& Peiró Velert, C. (2010). La colaboración científica en el campo de las Ciencias de la Actividad Física y el Deporte en España. Revista Española de Documentación Científica, 33(1), 90-105.

Vanderstraeten, R. (2011). Scholarly communication in education journals. Social Science History, 35(1), 109-130. doi: 10.1215/01455532-2010-018

Vinluan, L.R. (2012). Research productivity in education and psychology in the Philippines and comparison with ASEAN countries. Scientometrics, 91(1), 277-294. doi: 10.1007/s11192-0110496-5

Zawacki-Richter, O., Anderson, T., \& Tuncay, N. (2010). The Growing Impact of Open Access Distance Education Journals: A Bibliometric Analysis. The Journal of Distance Education, 24(3), 1.

Zich, I. (2011). Análisis bibliométrico de la revista "Bordón”. Bordón. Revista de pedagogía, 63(2), 141-152.

Zulueta, M.A. (2011). Metodología de los estudios bibliométricos. En J. López Yepes \& M.R. Osuna Alarcón (Eds.), Manual de Ciencias de la Información y Documentación (pp. 117-136). Madrid: Pirámide. 